\title{
The method of rock slope stability evaluation based on cloud model
}

\author{
Jie Gao ${ }^{1, a}$, Chunming $\mathrm{Qi}^{2, \mathrm{~b}}$, Xiaolin $\mathrm{Lei}^{3, \mathrm{c}}$ \\ ${ }^{1,2,3}$ College of Urban Construction, University of South China, Hengyang 421001, Hunan, China \\ a1013221067@qq.com, ${ }^{\mathrm{b}}$ qcm108100@sina.com, ${ }^{\mathrm{c}}$ 1092627545@qq.com
}

Keywords: cloud model;game theory; combination weighting;the slope stability; degree of certaint Abstract. According to the characteristics of the inevitable fuzziness and andomness in the slope stability evaluation,we proposed the model of rock slope stability evaluation based on cloud model, combined the combination weighting game theory considering subjective and objective weight,evaluate the slope instances. The comprehensive cloud of each factor on each stability level has been generated based on 7 indexes and evaluation standard.According the actual slope data,the degree of certainty for each slope on each stability level can be obtained. Finally, the maximum degree of certainty determined the slope stability level.Comparing the cloud model method with the grey correlation analysis and extension evaluation,proved that this evaluation result is reliable, the method is feasible and effective.

\section{Introduction}

As a natural geological body, the influence factors of slope is complexity and uncertainty. The impact of various factors are also different, understanding of people is still have limitations. So the boundary of slope stability and instability is not very clear actually,even very fuzzy ${ }^{[1-2]}$. Traditional slope stability analysis and evaluation methods mainly include the limit equilibrium method and numerical analysis method ${ }^{[3-5]}$.In recent years, some new theories are introduced by scholars,such as fuzzy comprehensive evaluation method ${ }^{[6-7]}$ 、 neural network evaluation method ${ }^{[8-9]}$,etc. This article is based on the fuzzy mathematics theory,starting from the non-linear relationship between slope's evaluation index and the level of stability,introduced the cloud model.We proposed the model of rock slope stability evaluation based on cloud model and combination weighting of game theory, Try to provide a new way of slope stability analysis.

\section{Introduction of cloud model}

Chinese academy of engineering Li De-yi proposed the concept of cloud model in 1995.Cloud model is a powerful tool to convert numerical quantitative analysis toconceptual qualitative analysis.Over the years,cloud model has been applied to different fields,such as comprehensive assessment of water quality 、 electronic information 、 simulation and prediction,etc,gained rather satisfactory achievement.

$\mathrm{U}=\{\mathrm{X}\}$.C is a qualitative concepts blong to $\mathrm{U}$. If quantitative value $\mathrm{X}$ belong to $\mathrm{U}$ and the function $\mathrm{X}$ attached $\mathrm{C}$ is $(0,1)$, it is the randomly generated value and has a certain tendency.So, each $\mathrm{X}$ can be regard as cloud droplets, their distribution make up the cloud: $u_{c}(x): U \rightarrow(0,1), \forall x \in U, x \rightarrow u_{c}(x)$. The relationship between one factor from $U$ to the membershipis of $\mathrm{C}$ is not a simple one to one but the one to many.

The basic cloud model is normal cloud model, normal distribution is general.Its digital characteristics use expectation、entropy and hyper entropy to characterize,reflect the qualitative concept and quantitative characteristics ${ }^{[10]}$ 。 Expectation: the most typical point in number field space, it's also the best point can reflect the qualitative concept,directly reflected by the center of the cloud. Entropy: reflects the area value of number field space that can be accepted by the qualitative concept,and the degree of cloud droplets belongs to the qualitative concept.Hyper entropy: reflects uncertainty of entropy, directly reflected by the size of cloud droplets cohesiveness. 


\section{The method of rock slope stability evaluation based on cloud model}

\section{Determine the evaluation standard and weight}

This article consider the membership of each factor on each stability level conforms with normal distribution.Evaluation standard come from $13^{\text {th }}$ literature,selected seven evaluation index, the slope stability are divided into five grades, the specific evaluation standard are shown in Table 1.

In slope stability evaluation,different evaluation indexes has different importance degree,so the weight is not the same.Usually,when we using a single way to get weight,it is hard to avoid the one-sidedness and arbitrariness.In order to overcome this defect,we should put the subjective and objective weights together then it can reflect the importance of the evaluation index integrallty ${ }^{[11]}$.So, we use the combination weighting game theory, algorithm is as follows ${ }^{[12]}$ :

Weights' linear combinations by $L_{\text {ways: }}$

$$
\omega=\sum_{k=1}^{L} a_{k} \bullet \omega_{k}^{T} ; \omega_{k}=\left(\omega_{k 1}, \omega_{k 2}, \ldots, \omega_{k n}\right),(k=1,2, \ldots, L) ;
$$

Optimized linear combination coefficients $a_{k}$, minimize the deviation:

$$
\min \left\|\sum_{k=1}^{L} a_{k} \omega_{k}-\omega_{k}\right\|_{2},(k=1,2, \ldots, L)
$$

Export: $\sum_{k=1}^{L} a_{k} \omega_{k} \omega_{k}{ }^{T}=\omega_{k} \omega_{k}{ }^{T}$ to get $\left(a_{1}, a_{2}, \ldots, a_{L}\right)$;Finally normalization get comprehensive weights $\omega^{*}$ :

$$
a_{k}^{*}=a_{k} / \sum_{k=1}^{L} a_{k} \omega^{*}=\sum_{k=1}^{L} a_{k} \omega_{k}^{T} ;(3)
$$

In this paper,the subjective weight using the analytic hierarchy process ${ }^{[13]}$ to get,objective weight using the entropy weight method ${ }^{[14]}$.The comprehensive weights $\omega=(0.047,0.350,0.037,0.149$, $0.100,0.067,0.238)$.

\section{Determine the parameters of cloud model}

$B_{\min }, B_{\max }$ is the maximum and minimum boundary of $V$, three digital characteristics of cloud: $E_{x}=\left(B_{\min }+B_{\max }\right) / 2 ; E_{n}=\left(B_{\max }-B_{\min }\right) / 6 ; H_{e}=k ; \mathrm{k}$ is a constant determined by experience. For a single boundary,we can determine default by upper and lower limit,then use the previous method to calculate.In Table2, a d represents boundary value.Such as

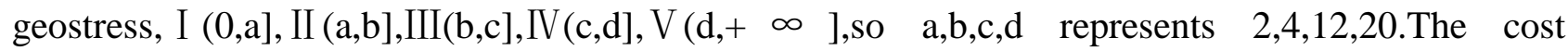

\begin{tabular}{|c|c|c|c|c|c|c|c|}
\hline Evaluation Index & $\begin{array}{l}\text { Rock Quality } \\
\text { Designation }\end{array}$ & $\begin{array}{l}\text { Rock Integrity } \\
\text { Designation }\end{array}$ & $\begin{array}{c}\text { Geostress / } \\
\mathrm{MPa}\end{array}$ & $\begin{array}{c}\text { Cohesion / } \\
\mathrm{MPa} \\
\end{array}$ & $\begin{array}{c}\text { Internal Frictional } \\
\text { Angle } /{ }^{\circ}\end{array}$ & $\begin{array}{c}\text { Slope } \\
\text { Height / m } \\
\end{array}$ & $\begin{array}{c}\text { Maximum Daily } \\
\text { Rainfall / mm }\end{array}$ \\
\hline Stable I & $\geq 90$ & $\geq 90$ & $\leq 2$ & $\geq 0.22$ & $\geq 37$ & $\leq 30$ & $\leq 20$ \\
\hline Relatively Stable II & 75 90 & 75 90 & $2 \sim 8$ & $0.12 \sim 0.22$ & $29 \sim 37$ & $30 \sim 45$ & $20 \sim 40$ \\
\hline Basically Stable III & $50 \sim 75$ & $50 \sim 75$ & $8 \sim 14$ & $0.08 \sim 0.12$ & $21 \sim 29$ & $45 \sim 60$ & $40 \sim 60$ \\
\hline Iinstability IV & $25 \sim 50$ & $30 \sim 50$ & $14 \sim 20$ & $0.05 \sim 0.08$ & $13 \sim 21$ & $60 \sim 80$ & $60 \sim 100$ \\
\hline Extremely Unstable V & $\leq 25$ & $\leq 30$ & $\geq 20$ & $\leq 0.05$ & $\leq 13$ & $\geq 80$ & $\geq 100$ \\
\hline
\end{tabular}
evaluation index according to $E_{x}, E_{n}$,otherwise according to $E_{x}^{\prime}, E_{n}^{\prime}$.

Table 1 The evaluation criteria of slope stability 


\begin{tabular}{cccc}
\hline Cloud & $E_{x}$ & $E_{n}$ & $H_{e}$ \\
\hline Stable I & $E_{x 1}=0 ; E_{x 1}{ }^{\prime}=d+c$ & $E_{n 1}=\left(E_{x 2}-E_{x 1}\right) / 3 ; E_{n 1}{ }^{\prime}=\left(E_{x 1}{ }^{\prime}-E_{x 2}{ }^{\prime}\right) / 3$ & 0.01 \\
Relatively Stable II & $E_{x 2}=(a+b) / 2 ; E_{x 2}{ }^{\prime}=(c+d) / 2$ & $E_{n 2}=\left(E_{x 2}-E_{x 1}\right) / 3 ; E_{n 1}{ }^{\prime}=\left(E_{x 2}{ }^{\prime}-E_{x 3}{ }^{\prime}\right) / 3$ & 0.01 \\
Basically Stable III & $E_{x 3} / E_{x 3}{ }^{\prime}=(b+c) / 2$ & $E_{n 3}=\left(E_{x 3}-E_{x 2}\right) / 3 ; E_{n 3}{ }^{\prime}=\left(E_{x 3}{ }^{\prime}-E_{x 4}{ }^{\prime}\right) / 3$ & 0.01 \\
Instability IV & $E_{x 4}=(c+d) / 2 ; E_{x 4}{ }^{\prime}=(a+b) / 2$ & $E_{n 4}=\left(E_{x 4}-E_{x 3}\right) / 3 ; E_{n 4}{ }^{\prime}=\left(E_{x 4}{ }^{\prime}-E_{x 5^{\prime}}\right) / 3$ & 0.01 \\
Extremely Unstable V & $E_{x 5}=d+c ; E_{x 5}{ }^{\prime}=0$ & $E_{n 5}=\left(E_{x 5}-E_{x 4}\right) / 3 ; E_{n 5}{ }^{\prime}=\left(E_{x 4}{ }^{\prime}-E_{x 5}{ }^{\prime}\right) / 3$ & 0.01 \\
\hline
\end{tabular}

\section{Generate the comprehensive cloud、determine the level of stability}

According to $E_{x}, E_{n}, H_{e}$,we use the cloud generator to get the comprehensive cloud based on seven evaluation index, see Figure 1(maximum daily rainfall is not shown in it). Taking slope height in figure 1 as an example,abscissa represents the slope height,ordinate represents the corresponding membership. The left to the right in the graph represent slope stability comprehensive cloud of level 1 to level 5.The figure left to right of geostress and maximum daily rainfall is from high level to low level,other instead.After determined the membership and multiplied by the index weight,where the maximum membership is the slope stability degree is.
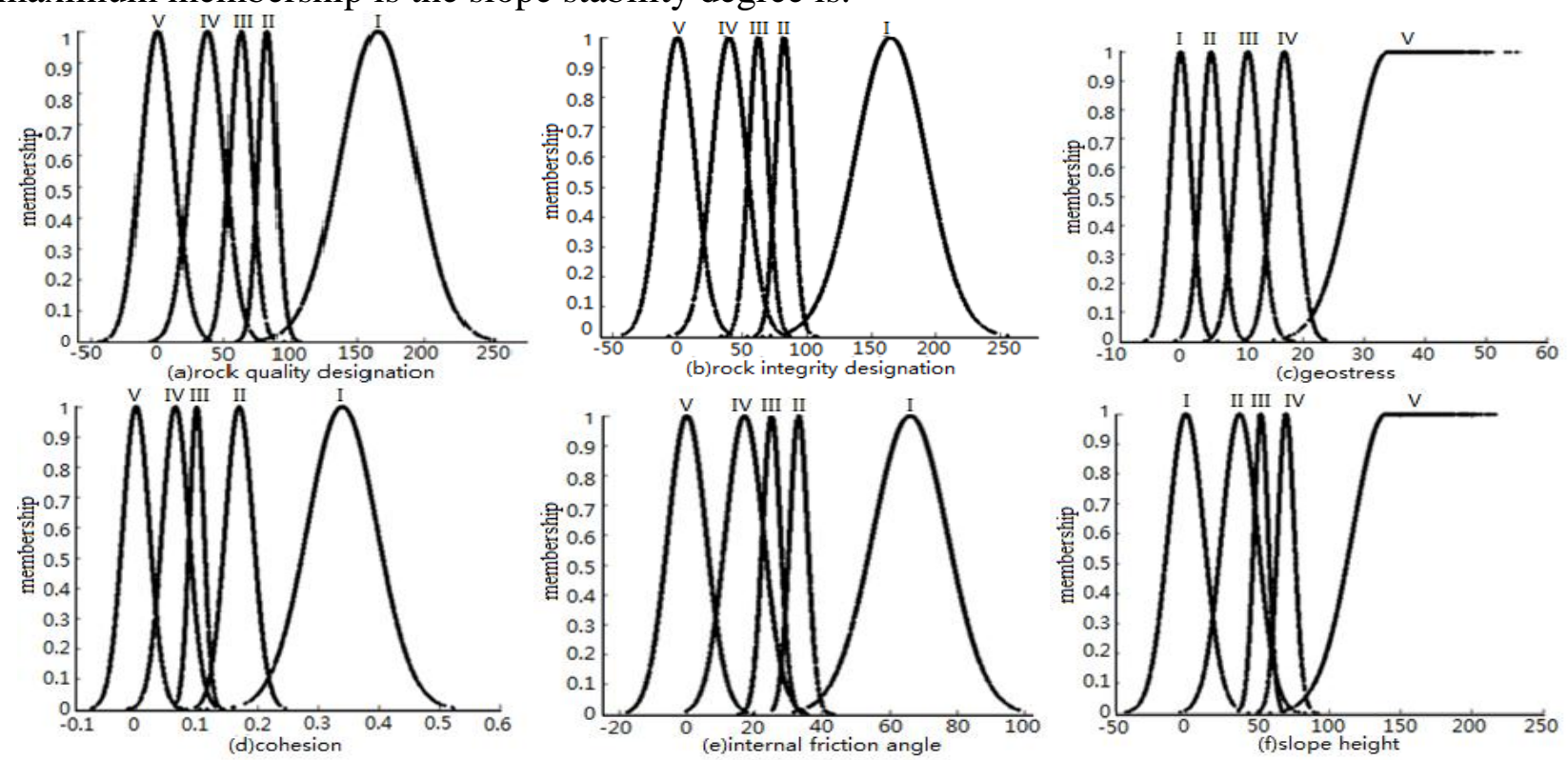

\section{Analysis of living example}

\section{Application example}

Take the four actual case of slope in $13^{\text {th }}$ literatureas research objects,measured values of slope indexes see Table 3. According to the principle of maximum membership degree,see the final evaluation results in Table 4. 
Table 3 Measured values of indexes for evaluation slopes

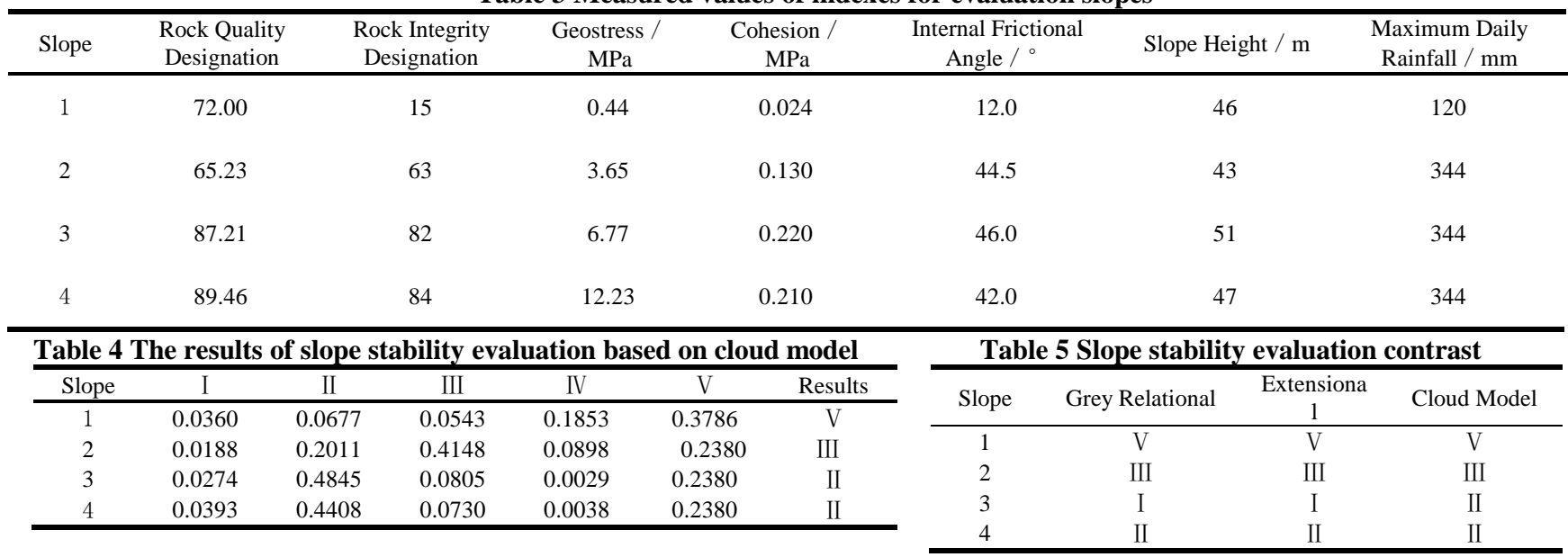

\section{Comparison and analysis}

Compared with other methods,comparison results in table 5.The cloud model method let membership of each factor on each stability level multiplied by the index weight then adds them to get the maximum degree of certainty. This reduces the impact on overall evaluation results caused by a single index on which level are fuzzy. Even if the values in final result is similar,according the principle of maximum membership degree to determine the level is still reasonable.

\section{Conclusion}

(1)Based on the fuzziness and randomness of slope stability evaluation and combined the combination weighting game theory considering subjective and objective weight,this article proposed the model of rock slope stability evaluation based on cloud model to evaluate slope of actual project.The results show that,cloud model method is feasible and effective, it provides a new method for slope stability evaluation.

(2)The study compared the cloud model method with grey relational analysis and extensional evaluation found the method in this paper is more considerate,the results is more reliable,verified the veracity of cloud model.

(3)It's hard to determine the three digital characteristics of cloud model.In this article,according the the method in table2 to determine $E_{x} 、 E_{n} 、 H_{e}$.Even so, I am also learning experience summed up by our predecessors then got table 2 .Therefore,it's very necessary to seek a more accurate method to determine the digital characteristics.

\section{References}

[1] Jianwen Huang, Jianlin Li, Yihong Zhou. Application of fuzzy analysis based on AHP to slope stability evaluation [J]. Chinese Journal of Rock Mechanics and Engineering, 2007, 26(Sup.1): 2627-2632. (in Chinese)

[2] Youcheng Zhai, Wengui Cao, Jiangying Wang,et al. Fuzzy evaluation method of slope stability based on uncertain aanalytic hierarchy process [J]. Rock and Soil Mechanics, 2011, 32(Sup.2): 539-543.

[3] Huaining Ruan.Applicationof comprehensive limit equilibrium theory in stability analysis of foundations and slopes[J].Journalof Hydraulic Engineering,1996,(3):46-55. (in Chinese)

[4] Hanhui Wang, Junxing Wang, Kaizhi Wang.Plastic limit analysis of slope stability using finiteelement[J].Rock and Soil Mechanics,2003,24(5):733-738. (in Chinese)

[5] Guo-qing Chen, Ruanqiu Hua, Hui Zhou,et al.Research on progressive failure for slope using dynamic strength reduction method[J].Rock and Soil Mechanics,2013,34(4):1140-1146. (in Chinese) 
[6] Quanmin Xie, Yuanyou Xia. Fuzzy hierarchy analysis on decision making of rockmass slope treatment based on entropy weight[J].Chinese Journal of Rock Mechanics and Engineering, 2003, 22(7):1117-1120. (in Chinese)

[7] Shigen Fu.Stability assessment of opencast mines slope based on fuzzy stochastic reliability[J]. Journal of Safety Science and Technology,2012,8(8):98-101. (in Chinese)

[8] Xiating Feng, Yongji Wang a, Shizong Lu.Neural network estimation of slope stability[J].Journal of Engineering Geology,1995,3(4):54-61. (in Chinese)

[9] Yuanyou Xia, Haifeng Xiong.Sensibility analysis of slope stability based on artificial neural network[J].Chinese Journal of Rock Mechanics and Engineering,2004,23(16):2703-2707. (in Chinese)

[10] Yanbing Gong.Comprehensive assessment on ecological risk of hexi corridor urbanizationbased on normal cloud model and entropy weight[J].Journal of Arid Land Resources and Environment, 2012,26(5):169-174. (in Chinese)

[11] Jialiang Chen.Research of performance evaluation method based on game theory[J].Fujian Computer,2003,9:15-16. (in Chinese)

[12] Zhiqing Zhang, Rui Chen, Chen Shen.Application of extension assessment method based on game theory evaluate the maintenance quality of urban rail transit subgrade[J].Journal of Wuhan university of technology,2012,36(4):691-694. (in Chinese)

[13] Lihong Ding.Research on estimation of slope stability based on improvedgrey correlation analysis and analytic hierarchy process[J].Rock and Soil Mechanics, 2011, 32(11):3437-3441. (in Chinese)

[14] Baodeng Hou, Jialei Li, Ni Pan,et al.Application of grey relevancy model based on ameliorated entropy for comprehensive evaluation of wetland environment qualit[J].Journal of Safety and Environmen,2008,8(6):80-83. (in Chinese) 